\title{
Predictive factors of clinical outcome in older surgical patients
}

\author{
Mario Bo*, Elena Cacello, Federica Ghiggia, \\ Laura Corsinovi, Francesca Bosco \\ Section of Geriatrics, Department of Medical and Surgical Disciplines, \\ Azienda Ospedaliera San Giovanni Battista-Molinette, \\ C.so Bramante 88, Turin 10126, Italy
}

Received 22 November 2005; received in revised form 5 May 2006; accepted 9 May 2006 Available online 25 July 2006

\begin{abstract}
We aimed to identify predictors of mortality and length of stay-in hospital in older surgical patients. In 294 patients (mean age $74.1 \pm 6.4$ years, $153 \mathrm{men}$ ), consecutively admitted to four surgery units of a university-teaching hospital to receive elective surgery (ES, 56.5\%) or urgent surgery (US, 43.5\%), the following variables were evaluated: demographics, clinical history (hypertension, diabetes mellitus (DM), coronary heart disease (CHD), heart failure (HF), cerebrovascular accidents, chronic obstructive pulmonary disease (COPD), active neoplasm, cognitive impairment, immobilization, pressure ulcers), physiopathology (Acute Physiology and Chronic Health Evaluation, APACHE, II), cognition/function (Short Portable Mental Status Questionnaire, SPMSQ; activity of daily living, ADL; instrumental activity of daily living, IADL), comorbidity (Cumulative Illness Rating Scale, CIRS, 1 and 2) and anesthesiology (American Score Anesthesiologist, ASA). The vital status of the patient at 1 month after discharge and the duration of hospitalization were recorded. One-month mortality rate was $6.1 \%$. Low hemoglobin and body mass index (BMI) values, increasing ASA score, and, only in US patients, ADL dependence and higher CIRS 1 score, were independently predictive of mortality. Low hemoglobin levels and, only in ES patients, higher CIRS 1 score were associated with prolonged hospitalization. Prognostic indicators specific to older people have limited value in mortality models in elderly surgical patients. (C) 2006 Elsevier Ireland Ltd. All rights reserved.
\end{abstract}

Keywords: Surgical mortality; Older surgical patients; Comorbidity

* Corresponding author. Tel.: +39011 6335328; fax: +39 0116961045.

E-mail address: mario.bo@unito.it (M. Bo).

0167-4943/\$ - see front matter (C) 2006 Elsevier Ireland Ltd. All rights reserved. doi:10.1016/j.archger.2006.05.007 


\section{Introduction}

The progressive aging of western populations, coupled with an increase in the actuarial life expectancy, is challenging the surgical and medical communities with an expanding group of patients who will require surgical interventions much later in life. Currently, the greatest demand for surgical procedures is in the elderly population (Vaitkevicus et al., 2003). Although chronologic age remains an independent risk for adverse surgical outcomes, the age associated reductions in the capacity to adapt to stress, a progression in functional frailty and the number of comorbid illnesses appear to be better predictors than age alone for poor surgical outcomes (Vaitkevicus et al., 2003). Studies exploring this issue showed that when comorbidity factors are considered into the analysis, age is no longer an independent predictor of poor outcome (Schrader et al., 1991; Sinanan et al., 2003). In addition to increasing operative risk, these factors also prolong recovery times, promote postoperative functional declines and increase the need for individual support after surgery. The American Society of Anesthesiologist Physical Status Classification (American Score Anesthesiologist-ASA) is routinely used to evaluate the risk of death in each patient who is candidate to surgery (Dripps et al., 1961; Owens et al., 1978). Several studies demonstrated that the ASA score is highly reliable also in older patients in different surgical settings (Hosking et al., 1989; Bufalari et al., 1996; El-Haddawi et al., 2002; Arenal and Bengoechea-Beeby, 2003). The Acute Physiology and Chronic Health Evaluation (APACHE) is another valid tool for the risk assessment in patients of a wide range of age, particularly in those who are candidate to emergent surgical procedures (Knaus et al., 1985; Giangiuliani et al., 1989; Barie et al., 1995; Koperna et al., 2001). On the other hand, there are very few studies, which investigated the impact on the surgical risk of those functional and cognitive variables, which have been shown to be highly predictive of clinical outcomes in older medical patients (Bo et al., 2003a,b). To our knowledge, there are no studies, which comprehensively investigated predictive factors of clinical outcomes in older patients undergoing elective surgery (ES) or urgent surgery (US) procedures. Knowledge of these predictors could prove useful to clinicians who have to estimate the global burden of surgical risk in older patients and to researchers who study the effectiveness of surgery on the risk of mortality in this setting. Inclusion of elderly specific variables could improve the prediction of clinical outcome beyond that achieved with currently available models, such as the ASA score or the APACHE II.

The aim of the present study was to identify predictive factors of mortality and length of stay-in hospital in a series of consecutive older patients admitted to a university-teaching hospital for US or ES under general anesthesia. For this purpose, an analysis was performed on clinical and demographic variables, including index of comorbidity and a number of parameters of physiological, functional and cognitive impairments investigated using standardized scales.

\section{Subjects and methods}

This prospective study consecutively recruited patients aged 65 and older who were admitted to four university surgical departments of a university-teaching hospital for US or 
ES under general anesthesia over a period of 5 months (from December 1, 2003 to May 1, 2004). Only patients admitted directly from the hospital first-aid unit were considered for the study; patients coming from other hospitals, from intensive care units or other departments within the hospital, and those who died or were discharged within $24 \mathrm{~h}$ of admission were excluded from the study. Two doctors from the staff of the Section of Geriatrics of the same hospital gathered the data using predefined forms within $24 \mathrm{~h}$ of patient admission. The following demographic variables were recorded: name and surname, sex, date of birth, age, education, marital status and living conditions (e.g., whether the patient was living alone, with a spouse, with his or her own children or relatives, with paid personnel, in long term facilities). The above information was obtained in part directly from the hospital admission form and in part from the patient and was always validated through an interview with relatives, caregivers or persons responsible for providing assistance. The clinical-history data were obtained from the clinical documentation produced at the time of admission and from the medical history gathered from the patient, whenever possible. Data obtained from surrogates were used in those patients who were unable to be interviewed. The clinical- and medical-history data were investigated with the aim of identifying the presence (before hospitalization) of Diabetes Mellitus (DM), hypertension, Coronary Heart Disease $(\mathrm{CDH})$, cerebrovascular accidents, Heart Failure (HF), Chronic Obstructive Pulmonary Disease (COPD), active neoplasm or history of cognitive-behavioral deterioration (prior clinical diagnosis of dementia). Patients were defined hypertensive when they were receiving blood pressure-lowering drugs, and diabetics when they had a prior diagnosis of diabetes or were treated with anti-diabetic medications. CHD was defined in the presence of clinical history of previous myocardial infarction or angina or coronary revascularization. HF and COPD were defined on the basis of clinical documentation of prior hospital admission with a diagnosis at discharge of HF and COPD, respectively, or in presence of instrumental evidence of an ejection fraction of less than $40 \%$ or a Forced Expiratory Volume in 1 second/Forced Expiratory Vital Capacity (FEV1/FVC) $<80 \%$, respectively. Further information were collected about the need for assistance from or supervision by a caregiver, immobilization or confinement to bed, use of nutritional aids by oral route or nutrition by parenteral or enteral route, and the need to use oxygen at home. The following data were gathered from the physical examination, which was performed upon patient admission and registered in the patient's clinical record: weight and height (from which the Body Mass Index (BMI), was calculated), body temperature in degrees centigrade, systolic and diastolic arterial pressure in $\mathrm{mmHg}$ in the recumbent position and heart rate and respiratory rate over $1 \mathrm{~min}$. The presence and severity of possible pressure ulcers was determined (scoring 0 , absence; 1 , skin redness; 2, involvement as far as the subcutis; 3 , muscle involvement; 4 , bone involvement).

In all patients, main blood chemistry examinations (serum creatinine, serum sodium, serum potassium, hematocrit, hemoglobin, while blood cell count) were performed upon arrival at the first-aid unit. Fasting serum albumin and total cholesterol values were collected the first morning after the patient's admission into the hospital ward. Blood-gas analysis of arterial blood was performed on all the patients in basal conditions at arrival in the hospital ward. Gathered data were used to fill in the APACHE II form. The APACHE II score is the sum of three scores: age points, chronic health points and acute physiology score points (Knaus et al., 1985). 
The data about functional and cognitive evaluation were obtained by applying the forms currently in use and previously validated for acceptance of patients into the care of the University's Geriatric Division (Fabris et al., 1989). Assessment of the functional status before the onset of the disease that caused the hospital admission was performed using activity of daily living (ADL) (Katz et al., 1963) and the instrumental activity of daily living (IADL) (Lawton and Brody, 1969) scales. Data on ADL and IADL were obtained from the patient, whenever possible, and integrated with information gathered from a close relative, caregiver or person responsible for providing assistance. The cognitive status of the patient upon admission was assessed using the Short Portable Mental Status Questionnaire (SPMSQ). Surrogate responses were used in cognitively impaired patients (SPMSQ $\geq 4$ ) (Pfeiffer, 1975). The Cumulative Illness Rating Scale (CIRS) has been used to evaluate concomitants diseases; the index of severity (CIRS 1) and the index of comorbidity (CIRS 2) were assessed (Linn et al., 1968).

The suspected diagnosis at admission and the type of surgery (US or ES) was defined by the surgeon on the basis of medical history, thorough physical examination and instrumental examinations. Cases for which surgery was indicated and was performed within $24 \mathrm{~h}$ from admission were considered US; cases which were not included as US were considered ES. The anesthesiology evaluation was performed by qualified anesthesiologists according to the ASA scale (Dripps et al., 1961; Owens et al., 1978), which defines five levels of surgical risk. Typology of surgical intervention and definite diagnosis were gathered from medical charts and from surgical record, according to the International Classification of Diseases, Ninth Revision, Clinical Modification. The outcome of the hospitalization was obtained from the hospital discharge summary. The following variables were considered: vital status of the patient (whether alive or dead) upon discharge and 1 month after discharge, and duration of hospitalization. The vital status of all patients 1 month after discharge has been evaluated through telephonic interview with patients themselves or their relatives or persons responsible for their assistance.

Primary clinical outcome was vital status 1 month after discharge; secondary clinical outcome was duration of hospitalization. Analysis was performed on the overall sample of patients and separately on patients who received ES or US procedures.

The normal distribution of the quantitative variables was evaluated using a graphical method and both the Kolmogorov-Smirnov test and the Shapiro-Wilk test. The distribution was considered Gaussian when the probability associated to both tests was high $(p<0.01)$. Quantitative variables have been described as mean, median, standard deviation and interquartile range. Using univariate analysis, the variables associated with clinical outcomes were identified: the dichotomous variables were analyzed using contingency tables with Chi-square test and odds ratio (OR) with a 95\% confidence interval (CI), whereas the parametric continuous variables were analyzed using the Student's $t$-test. The length of stay-in hospital was analyzed through determination of the $25^{\circ}$ percentile of the duration of hospitalization and comparison between groups below and over this value. The indices of functional impairment (ADL, IADL) and cognitive impairment (SPMSQ) were evaluated as continuous variables and in terms of dichotomous parameters. Patients with ADL score $\geq 1$ or IADL $\leq 5$ were considered functionally dependent. Patients with SPMSQ score $\geq 4$ were considered cognitively impaired. The level of significance used was $p<0.05$. Significant variables were then introduced into the multivariate analysis by 
means of logistic regression using the stepwise-forward method, to identify variables independently associated with each clinical outcome.

\section{Results}

During the period under examination, 452 patients aged 65 and older were admitted to a general surgery unit from the first-aid of the hospital; 148 subjects were not included in the study because they did not receive surgery or because they received regional anesthesia. Ten of the 304 eligible patients were not considered for analysis because of missing data. Two-hundred ninety-four patients (mean age $74.1 \pm 6.4$ years, range $65-94 ; 153$ men) were recruited for the study. ES and US procedures were performed, respectively, in 166 $(56.5 \%)$ and $128(43.5 \%)$ patients. In $52.4 \%$ of patients, the disease responsible for hospital admission and surgical intervention was a neoplasm. The most frequent diagnosis among patients who received ES was a malignant gastrointestinal disorder. Among patients who underwent US, the most common diseases were intestinal perforation, acute mesenteric ischemia, acute cholecystitis and acute pancreatitis.

Table 1 summarizes the main characteristics of the 294 patients enrolled. Functional dependence was present in $12.2 \%$ of patients according to the ADL scale and in $14.6 \%$ according to the IADL scale. Mean CIRS 1 and CIRS 2 scores were $3.0 \pm 0.5$ and $2.4 \pm 1.3$, respectively. Mean APACHE II score was $11.0 \pm 3.2$ and mean ASA score was $2.6 \pm 0.8$.

Two-hundred eighty-two patients $(95.9 \%)$ were discharged alive. In-hospital mortality rate was $7.8 \%$ among patients who received US and $1.2 \%$ among those who received ES. At 1-month follow-up, 6 of 282 patients who were discharged alive had died, giving a total

Table 1

Demographic, anamnestic, and clinical characteristics of the population examined $(n=294)$

\begin{tabular}{ll}
\hline Male, $n(\%)$ & $153(52.0)$ \\
Age (years) & $74.1 \pm 6.4$ \\
Hemoglobin $(\mathrm{g} / \mathrm{dl})$ & $12.2 \pm 2.1$ \\
BMI $\left(\mathrm{kg} / \mathrm{m}^{2}\right)$ & $24.5 \pm 4.5$ \\
Serum albumin $(\mathrm{g} / \mathrm{dl}$, normal range 3.5-5.2) & $3.4 \pm 0.6$ \\
APACHE II (score) & $11.0 \pm 3.2$ \\
ASA (score) & $2.6 \pm 0.8$ \\
ADL, functions lost & $0.8 \pm 1.6$ \\
IADL, functions preserved & $10.4 \pm 3.9$ \\
SPMSQ, errors & $2.8 \pm 2.9$ \\
CIRS 1 (score) & $3.0 \pm 0.5$ \\
CIRS 2 (score) & $2.4 \pm 1.3$ \\
Hypertension, $n(\%)$ & $175(59.5)$ \\
DM, $n(\%)$ & $39(13.3)$ \\
HF, $n(\%)$ & $20(6.8)$ \\
CHD, $n(\%)$ & $34(11.6)$ \\
COPD, $n(\%)$ & $23(7.8)$ \\
Active neoplasm, $n(\%)$ & $142(48.3)$ \\
History of cognitive deterioration, $n(\%)$ & $8(2.7)$ \\
\hline
\end{tabular}

Data are presented as mean \pm Standard Deviation (S.D.) unless otherwise indicated. 
Table 2

Variables associated with 1-month mortality in the overall sample of patients: univariate analysis

\begin{tabular}{|c|c|c|c|c|}
\hline Variables & Alive & Dead & & $p$ \\
\hline BMI $\left(\mathrm{kg} / \mathrm{m}^{2}\right)$ & $24.9 \pm 4.4$ & $22.9 \pm 3.8$ & $2.719^{\mathrm{a}}$ & 0.007 \\
\hline Hemoglobin (g/dl) & $12.3 \pm 2.1$ & $11.3 \pm 2.1$ & $4.140^{\mathrm{a}}$ & $<0.001$ \\
\hline Serum albumin $(\mathrm{g} / \mathrm{dl})$ & $3.5 \pm 0.6$ & $3.0 \pm 0.9$ & $2.520^{\mathrm{a}}$ & 0.012 \\
\hline ADL (score) & $0.4 \pm 1.2$ & $0.8 \pm 1.8$ & $-3.600^{\mathrm{a}}$ & $<0.001$ \\
\hline IADL (score) & $12.4 \pm 2.9$ & $10.6 \pm 4.1$ & $2.028^{\mathrm{a}}$ & 0.043 \\
\hline SPMSQ (score) & $1.5 \pm 1.5$ & $2.6 \pm 1.9$ & $-2.246^{\mathrm{a}}$ & 0.026 \\
\hline APACHE II (score) & $10.9 \pm 3.3$ & $12.5 \pm 3.1$ & $-2.582^{\mathrm{a}}$ & 0.010 \\
\hline ASA (score) & $2.6 \pm 0.8$ & $2.9 \pm 0.7$ & $-2.562^{\mathrm{a}}$ & 0.011 \\
\hline CIRS 1 (score) & $3.0 \pm 0.4$ & $3.2 \pm 0.6$ & $-2.746^{\mathrm{a}}$ & 0.006 \\
\hline $\mathrm{ADL} \geq 1$ (dependent) & $31 / 276$ & $4 / 18$ & $16.763^{\mathrm{b}}$ & $<0.001$ \\
\hline IADL $\leq 5$ (dependent $)$ & $39 / 276$ & $5 / 18$ & $14.339^{\mathrm{b}}$ & $<0.001$ \\
\hline SPMSQ $\geq 4$ (cognitively impaired) & $40 / 276$ & $4 / 18$ & $7.833^{\mathrm{b}}$ & 0.005 \\
\hline
\end{tabular}

number of 18 deaths $(6.1 \%)$. Of the 282 patients who were discharged alive, $15(5.3 \%)$ were discharged in long-term facilities or nursing-homes: this type of discharge was more common among patients who received US (7.0\%) than in those who underwent ES (3.6\%). Mean length of stay-in hospital was $14.8 \pm 10$ days.

Table 2 shows the variables associated with 1-month mortality in the overall sample of patients. After multivariate analysis, low hemoglobin levels, low BMI and increasing ASA score were independently predictive of mortality (Table 3 ). The extremely low mortality rate observed among patients who received ES did not consent identification of predictive factors in this group of patients. Low hemoglobin and BMI values, ADL functional dependence, and increasing ASA and CIRS 1 scores were found to be independently predictive of mortality among US patients (Table 4).

Table 3

Variables associated with 1-month mortality in the overall sample of patients: multivariate analysis

\begin{tabular}{lllll}
\hline Variables & $\beta$ & \pm S.E. & $p$ & $95 \%$ CI \\
\hline Hemoglobin $(\mathrm{g} / \mathrm{dl})$ & -0.369 & 0.16 & 0.021 & $0.506-0.946$ \\
ASA $(\mathrm{score})$ & 0.467 & 0.213 & 0.029 & $1.050-2.423$ \\
BMI $\left(\mathrm{kg} / \mathrm{m}^{2}\right)$ & -0.161 & 0.071 & 0.02 & $0.741-0.977$ \\
\hline
\end{tabular}

Table 4

Variables associated with 1-month mortality in patients who underwent US: multivariate analysis

\begin{tabular}{lrlll}
\hline Variables & \multicolumn{1}{c}{} & \pm S.E. & $p$ & $95 \%$ CI \\
\hline Hemoglobin $(\mathrm{g} / \mathrm{dl})$ & -0.380 & 0.138 & 0.006 & $0.522-0.897$ \\
ASA $(\mathrm{score})$ & 0.476 & 0.206 & 0.021 & $1.075-2.411$ \\
BMI $\left(\mathrm{kg} / \mathrm{m}^{2}\right)$ & -0.140 & 0.069 & 0.044 & $0.759-0.996$ \\
CIRS 1 (score) & 1.198 & 0.607 & 0.049 & $1.007-10.890$ \\
ADL (dependence) & -1.660 & 0.582 & 0.004 & $0.061-0.595$ \\
\hline
\end{tabular}


In the overall sample, only low hemoglobin levels were significantly associated with greater duration of hospitalization $(\beta-0.474, p<0.001)$. Among patients who received ES, low hemoglobin levels $(\beta-0.222, p=0.045)$ and increased CIRS 1 score $(\beta 1.697$, $p=0.002)$ were independently predictive of prolonged length of stay-in hospital.

\section{Discussion}

To our knowledge, very few studies investigated comprehensively predictors of clinical outcomes among older surgical patients. The results of the present study indicate that in a comprehensive evaluation of variables associated with postoperative mortality in older patients, measures of functional and cognitive status as well as index of comorbidity have little impact on mortality. In the overall sample of patients, only low hemoglobin and BMI values, beyond ASA score, were independent predictors of 1-month mortality. However, among patients who received US, also functional dependence and greater severity of comorbidities were independently predictive of a higher risk of 1-month mortality.

The mortality rates observed in the present study in the overall sample and among patients who underwent US procedures are substantially in accordance with other studies (Hosking et al., 1989; Bufalari et al., 1996; Polanczyk et al., 2001; Roteano et al., 2002). However, the mortality rate observed among patients who received ES is near the lowest reported in previous studies (Goffi et al., 1999; Polanczyk et al., 2001; Roteano et al., 2002). Several factors might account for this extremely low mortality rate, including a more cautious selection of patients candidate to surgery, a less severe degree of pathology and, finally, the high surgical volumes and the availability of multidisciplinary competences in a universityteaching hospital. As it was expected, mortality rate was remarkably higher among patients who underwent US, than in patients who received ES. US is by itself a well-defined predictor of mortality at any age (Hosking et al., 1989; Berger et al., 1992; Bufalari et al., 1996; ElHaddawi et al., 2002; Arenal and Bengoechea-Beeby, 2003).

This study differs from other studies of mortality in surgical older patients in that it aimed to investigate predictors of mortality among a wide series of variables, including conventional scores (ASA and APACHE II), anamnestic and clinical variables, measures of functional and cognitive status, and presence and severity of comorbid illnesses. Similarly to other studies (Bufalari et al., 1996; Polanczyk et al., 2001; Roteano et al., 2002; Arenal and Bengoechea-Beeby, 2003), we observed that age is not predictive of mortality. Studies that evaluated mortality in patients aged 65 and older concluded that, in absence of functional limitations, older age by itself does not involve a greater risk of death (Bufalari et al., 1996; Vaitkevicus et al., 2003). We found that both low hemoglobin levels and BMI values are predictive of mortality in the overall sample and in US patients. Low levels of both these variables are likely surrogates of poor global health (Wolters et al., 1997; Roteano et al., 2002; Bo et al., 2003a; Dos Santos Junqueira et al., 2003). In patients who received US procedures, both functional dependence (ADL) and greater severity of comorbid illnesses (CIRS 1) were further independent predictors of mortality. Although the functional impairment detected by the ADL and IADL scales was predictive of unfavorable prognosis by univariate analysis, only ADL dependence proved to be independently predictive of mortality. These findings likely reflect the different domains 
explored by these two scales. Indeed, loss of independence in ADLs usually indicates greater frailty of the patient than loss of independence in IADLs. Older patients are usually affected by several concurrent diseases: therefore it is not unexpected that the severity rather than the number of comorbid illnesses may better identify the most frail individuals, with a greater risk of postoperative mortality. On the contrary, it was somehow unexpected that APACHE II score did not prove to be predictive of mortality in US patients. Although the mean APACHE II score in our study $(12.7 \pm 2.8)$ is in accordance with that reported in previous studies (Bernstein and Offenbartl, 1991; Berger et al., 1992; Goffi et al., 1999; Koperna et al., 2001), the narrow range of APACHE II scores of our sample (7-23) might have reduced the ability to detect a predictive role.

As a whole, our findings show that the evaluation of prognostic indicators specific to older people have limited value in mortality models in elderly surgical patients. Technical advances in perioperative care and in the technology of surgery are thought to have improved both the safety and efficacy of surgical procedures in the elderly population, especially in the very old. Yet, determination of few variables (hemoglobin, BMI, functional status and severity of comorbidities) might add to the evaluation of the risk of death through the conventional ASA score in older patients who are candidate to US. However, the inclusion of these few variables in the risk stratification may provide limited benefit in patients candidate to US. Indeed, when a patient has an immediate need for surgery, with a poor likelihood of survival without it, there is very narrow room both for a detailed risk-benefit evaluation and for an attempt of clinical optimization. It can be hypothesized that these factors might play a much more important role in predicting functional outcomes after surgery in older patients.

In a period of limited resources in several hospital settings, the duration of the hospitalization is an issue of relevant interest for surgeons and for hospital managers. In this view, elderly patients are considered at "high risk" of prolonged stay-in hospital because of an increased number of complications and more problematic discharges. We found that, among patients who received ES, low hemoglobin levels and greater severity of comorbid illnesses were associated with a prolonged hospitalization. These variables identify the most frail and compromised patients who, even in the setting of ES, are most prone to develop perioperative complications and have greater risk of prolonged hospitalization. It can be speculated that a more aggressive perioperative medical and rehabilitative approach in these patients might result in a reduced risk of complications and a shorter duration of hospitalization. However, this preoperative approach could not be applied to patients candidate to US; therefore, we did not perform such analysis to individuate predictors of length of stay-in hospital among patients who received US.

Some limitations of this study must be addressed. The sample of patients investigated consisted of older patients admitted to different surgical units in a university-teaching hospital; therefore, our results should not be generalized to patients hospitalised at other institutions. Despite the single-site design of the study and the relatively small sample of patients investigated, to our knowledge this is the first study aimed to a comprehensive evaluation of the risk of death in older surgical patients. Although there is little evidence that regional anesthesia may decrease perioperative risk, at least in relatively healthy patients of any age, the choice of anesthetic technique may pose the older patients to different risk of complications in the perioperative period. Therefore, it was decided to 
enrol only patients who received general anesthesia, in order to avoid a confounding factor which by itself can heavily affect clinical outcomes in older surgical patients.

In conclusion, the ASA score remains an excellent predictor of mortality in older surgical patients, whereas prognostic indicators specific to older people have limited value in mortality models in these patients. Measures of clinical wasting (low hemoglobin and BMI values) and, only in candidates to US procedures, functional dependence and severity of comorbid illnesses, are additional predictors of mortality. Low hemoglobin values and severity of comorbid diseases are independent predictors of duration of stay-in hospital. Further studies are needed to evaluate the impact of these variables on functional outcomes after surgery in older patients.

\section{References}

Arenal, J.J., Bengoechea-Beeby, M., 2003. Mortality associated with emergency abdominal surgery in the elderly. Can. J. Surg. 46, 111-116.

Barie, P.S., Hydo, L.J., Fischer, E., 1995. Comparison of APACHE II and III scoring system for mortality prediction in critical surgical illness. Arch. Surg. 130, 77-82.

Berger, M.M., Marazzi, A., Freeman, J., Chiolero, R., 1992. Evaluation of the consistency of Acute Physiology Score and Chronic Health Evaluation (APACHE II) scoring in a surgical intensive care unit. Crit. Care Med. 20, 1681-1687.

Bernstein, G.M., Offenbartl, S.K., 1991. Adverse surgical outcomes among patients with cognitive impairment. Am. Sur. 57, 682-690.

Bo, M., Massaia, M., Raspo, S., Bosco, F., Cena, P., Molaschi, M., Fabris, F., 2003a. Predictive factors of inhospital mortality in older patients admitted to a medical intensive care unit. J. Am. Geriatr. Soc. 51, 529533.

Bo, M., Raspo, S., Massaia, M., Cena, P., Bosco, F., Fabris, F., Antro, C., Gai, V., 2003b. A predictive model of inhospital mortality in elderly patients admitted to a medical intensive care unit. J. Am. Geriatr. Soc. 51, 15071508.

Bufalari, A., Ferri, M., Cao, P., Cirocchi, R., Bisacci, R., Moggi, L., 1996. Surgical care in octagenarians. Br. J. Surg. 83, 1783-1787.

Dos Santos Junqueira, J.C., Cotrim Soares, E., Rodrigues Correa Filho, H., Fenalti Hoehr, N., Oliveira Magro, D., Ueno, M., 2003. Nutritional risk factors for postoperative complications in Brazilians elderly patients undergoing major elective surgery. Nutrition 19, 321-326.

Dripps, R.D., Lamont, A., Eckenoff, J.E., 1961. The role of anaesthesia in surgical mortality. J. Am. Med. Assoc. $178,261-266$.

El-Haddawi, F., Abu-Zidan, F.M., Jones, W., 2002. Factors affecting surgical outcome in the elderly of Auckland hospital. ANZ J. Surg. 72, 537-541.

Fabris, F., Macchione, C., Molaschi, M., Ferrario, E., Pernigotti, L., Visentin, P., 1989. The clinical geriatric chart: a proposal for multidimensional functional assessment. Minerva Med. 80, 1-56.

Giangiuliani, G., Mancini, A., Gui, D., 1989. Validation of a severity illness score (APACHE II) in a surgical intensive care unit. Intensive Care Med. 15, 519-522.

Goffi, L., Saba, V., Ghiselli, R., Decozione, S., Mattei, A., Carle, F., 1999. Perioperative APACHE II and ASA score in patients having major surgical operations: prognostic value and potential clinical application. Eur. J. Surg. 165, 730-735.

Hosking, M.P., Warner, M.A., Lobdell, C.M., Offord, K.P., Melton, L.J., 1989. 3rd Outcome of surgery in patients 90 years of age and older. J. Am. Med. Assoc. 261, 1909-1915.

Katz, S., Ford, A.B., Moskowitz, R.W., Jackson, B.A., Jaffe, M.W., 1963. Studies of illness in the aged. The index of ADL, a standardized measure of biological and psychosocial function. J. Am. Med. Assoc. 185, 914-919.

Knaus, W.A., Draper, E.A., Wagner, D.P., Zimmerman, J.E., 1985. APACHE II: a severity of disease classification system. Crit. Care Med. 13, 818-829. 
Koperna, T., Semmler, D., Marian, F., 2001. Risk stratification in emergency surgical patients: is the APACHE II score a reliable marker of physiological impairment? Arch. Surg. 136, 55-59.

Lawton, M.P., Brody, E.M., 1969. Assessment of older people: self-maintaining and instrumental activities of daily living. Gerontologist 9, 179-186.

Linn, B.S., Linn, M.W., Gurel, L., 1968. Cumulative Illness Rating Scale. J. Am. Geriatr. Soc. 16, 622-626.

Owens, W.D., Felts, J.A., Spitznagel Jr., E.L., 1978. ASA physical status classifications: a study of consistency of rating. Anaesthesiology 49, 239-243.

Pfeiffer, E., 1975. A Short Portable Mental Status Questionnaire for the assessment of organic brain deficit in elderly patients. J. Am. Geriatr. Soc. 23, 433-441.

Polanczyk, C.A., Marcantonio, E., Goldman, L., Rohde, L.E., Orav, J., Mangione, C.M., Lee, T.H., 2001. Impact of age on perioperative complications and length of stay in patients undergoing noncardiac surgery. Ann. Intern. Med. 134, 637-643.

Roteano, M., Calligaris, L., Pozzetto, B., Cibi, N., Bortul, M., 2002. Evaluation of surgical risk in elderly patients: a review of 207 cases. Chirurgia Italiana 54, 437-445.

Schrader, L.L., Mc Millen, M.A., Watson, C.B., MacArthur, J.D., 1991. Is routine preoperative hemodynamic evaluation of nonagenarians necessary? J. Am. Geriatr. Soc. 39, 1-5.

Sinanan, M., Kao, L., Vedovatti, P.A., 2003. Surgery in the elderly population. In: Hazzard, W.R., Blass, J.P., Halter, J.B., Ouslander, J.G., Tinetti, M.E. (Eds.), Principles of Geriatric Medicine and Gerontology. fifth ed. Mc Graw Hill, New York, pp. 385-399.

Vaitkevicus, V.P., Kirsh, M.M., Orringer, M.B., 2003. Perioperative evaluation and management. In: Hazzard, W.R., Blass, J.P., Halter, J.B., Ouslander, J.G., Tinetti, M.E. (Eds.), Principles of Geriatric Medicine and Gerontology. fifth ed. Mc Graw Hill, New York, pp. 361-371.

Wolters, U., Wolf, T., Stutzer, H., Schroder, T., Pichlmaier, H., 1997. Risk factors, complications, and outcome in surgery: a multivariate analysis. Eur. J. Surg. 163, 563-568. 